\title{
The relationship between behavioural problems in preschool children and parental distress after a paediatric burn event
}

\author{
Anne Bakker • Peter G. M. van der Heijden • \\ Maarten J. M. van Son - Rens van de Schoot • \\ Els Vandermeulen • Ann Helsen • Nancy E. E. Van Loey
}

Received: 8 April 2013/Accepted: 8 January 2014

(c) Springer-Verlag Berlin Heidelberg 2014

\begin{abstract}
This study examines mother- and father-rated emotional and behaviour problems in and worries about 0 - to 5-year-old children at 3 and 12 months after a burn event and the relation with parental distress. Mothers $(n=150)$ and fathers $(n=125)$ representing 155 children participated in this study. Child emotional and behaviour problems and parental worries about the child were assessed with the Child Behavior Checklist at both time points. Parents' level of acute subjective distress was assessed within the first month after the burn event with the Impact of Event Scale. Mothers and fathers held comparable views of their child's emotional and behaviour problems, which were generally within the normal limits. Parents' own acute stress reactions were significantly related to parent-rated child behaviour problems at 3 and 12 months postburn. A substantial part of mothers'
\end{abstract}

A. Bakker $(\bowtie) \cdot$ N. E. E. Van Loey

Dutch Burn Centres, P. O. Box 1015, 1940 EA Beverwijk,

The Netherlands

e-mail: abakker@burns.nl

P. G. M. van der Heijden - R. van de Schoot

Department of Methodology and Statistics, Faculty of Social

Sciences, Utrecht University, Utrecht, The Netherlands

M. J. M. van Son · N. E. E. Van Loey

Department of Clinical and Health Psychology,

Utrecht University, Utrecht, The Netherlands

R. van de Schoot

Optentia Research Program, Faculty of Humanities,

North-West University, Vanderbijlpark, South Africa

E. Vandermeulen

Military Hospital Neder-over-Heembeek, Brussels, Belgium

A. Helsen

University Hospital Leuven, Leuven, Belgium and fathers' worries about the child concerned physical and emotional aspects of the burn trauma, and potential future social problems. Parents with high acute stress scores more often reported burn-related concerns about their child at 3 and 12 months postburn. Health-care professionals should be informed that parents' distress in the subacute phase of their child's burn event may be related to subsequent worries about their child and to (parent-observed) child emotional and behaviour problems. The authors recommend a family perspective, with particular attention for the interplay between parents' distress and parent-reported child behaviour problems and worries, in each phase of paediatric burn care.

Keywords Burns · Children · Behavioural problems . Parental distress - Fathers - Interparental agreement

Burns are among the most common causes for injuries in children [1], and in particular children under the age of 5 are at increased risk to sustain burn injuries [2]. Rapid motor and cognitive development during early childhood contribute to a high incidence of predominantly scald burns in this young population [2, 3]. Commonly reported mechanisms for scald burns are for instance reaching for a cup on the edge of a counter, a pan on a stove, or pulling a table cloth [4]. Because children have a thinner and therefore more vulnerable skin than adults, seemingly modest accidents with hot fluids may result in severe burns with permanent scarring. Burns have been described as one of the most serious and traumatic injuries a child may experience $[5,6]$. The potential life-threatening character of the injuries, pain, hospitalisation and/or frequent visits to the hospital, and aspects of the medical treatment, such as repeated wound dressing changes, scar management, and in some cases skin grafting procedures are significant 
additional stressors. In the longer run, rehabilitation and reconstructive surgeries may be necessary. Permanent alterations to the child's appearance, functional limitations, and issues in social life may be further challenges for children [6] and may elicit parental worries about the future of their child [7].

Serious medical events, such as burn injuries, may evoke a range of psychological reactions in children [8]. Previous studies in preschool children with burns have described externalising as well as internalising difficulties. Mothers reported for instance, by means of a semi-structured interview, that disturbed behaviours, such as anxiety and increased temper tantrums, were present in $56 \%$ of hospitalised preschool children [9], and $16 \%$ of preschool children met criteria for newly diagnosed separation anxiety disorder and oppositional defiant disorder at 6 months postburn as measured with a diagnostic interview with the primary caregiver [6]. Traumatic stress symptomatology was present in 25-29\% in the first months [6, 10], and in $13 \%$ of preschool children at an average of 1 year postburn [11]. In a Dutch-Belgian study on child internalising problems conducted during the child's hospitalisation, $25 \%$ of 2- to 14-year-old children displayed problems in the clinical range [12] and a US study found significantly higher levels of internalising problem behaviour than a normative sample at an average of 1 year postburn [13]. Differently, a prospective study did not find significant group differences compared to reference data [14], and a cross-sectional study at 1 year postburn on average found internalising problems and externalising problems only in 5 and $3 \%$ of preschool children, respectively [11]. Explanations for the discrepancy in findings may include variety in study outcomes, measures, timeframe, and sample characteristics (e.g. severity of injuries, socioeconomic background).

So far, no studies prospectively followed children beyond the first 6 months postburn. Moreover, study samples were relatively small ( $n$ 's between 17 and 76), with the exception of one Australian study on 130 young burn survivors with minor burn injuries [6]. Further, mothers were the principal informants on child postburn emotional and behavioural outcome in virtually all paediatric burn studies [15]. Moreno and colleagues [16] have argued that "the scarcity of father involvement in psychological research has likely yielded an incomplete picture of the familial context involved in child and adolescent psychopathology, especially in the assessment of youth's internalising and externalising behaviour problems" ( $p$. 915). Mothers and fathers may hold a different view of their child's behaviour [16], although it has been found that mothers and fathers overall show moderate to high levels of agreement concerning their child's internalising and externalising behaviour problems [17]. Up to now, studies on children with burns did not address the degree of interparental agreement or discrepancy on ratings of child internalising and externalising behaviour problems.

Paediatric medical events may also seriously affect the parents and a relational model on psychotrauma in early childhood has presented patterns of bidirectional relations between family members' symptoms [18]. Previous studies have reported considerable rates of depression [19], anxiety [20], acute stress symptoms [21, 22], and posttraumatic stress [23, 24]. Moreover, studies found that many mothers had concerns about their child's health, scarring and appearance, and social issues after a burn event [7, 20]. Mothers' emotional reactions were associated with their child's reactions after the burn event in several studies [10, $11,25]$. Information on emotional reactions and worries of fathers is very limited $[11,21,22,26]$. The few available studies have shown that within couples mothers may experience more stress symptoms than fathers [21, 26], but fathers are unquestionably affected by their child's burn event as well. So far, only one study specifically investigated the mutual relationship between father and child postburn outcome [11]. This study among parents of 1- to 4-year-old children found that mothers' but not fathers' PTSD symptoms in the aftermath of the child's burn event were related to the child's symptoms. However, mothers were the single informant to report on child posttraumatic stress and behavioural functioning in that study. The authors suggested that the absent relationship between the father's PTSD symptoms and the child's outcome might in fact be due to the single use of the mother's rating of the child's functioning [11]. Literature on other child traumatic events [27, 28] and child psychopathology [29] has showed somewhat mixed results, but in general both mothers' and fathers' psychological functioning were associated with child emotional and behavioural problems. Up to now, it is unclear to what extent fathers' reactions are related to his preschool child's postburn reactions.

In summary, a burn event is a potentially traumatic event for both children and their parents and there is a lack of prospective research that extends beyond the period of hospitalisation. In addition, the traditional research focus on mothers limits our understanding of the father's perspective on child functioning, his worries, and father-child associations in terms of postburn outcome. The first aim of this study was to study child behaviour problems at 3 and 12 months after a paediatric burn event. Compared to reference data, children with burns were hypothesised to show elevated levels of behavioural problems at 3 months postburn. Problem behaviour was in particular expected in the internalising domain, as this was previously found in other paediatric burn studies [11-13]. At 12 months postburn, the level of problems was expected to have significantly decreased, pointing at recovery within the first year 
postburn. Second, we hypothesised that mothers and fathers would show a moderate to high level of correspondence in terms of their rating of internalising and externalising problem behaviour in the child. Third, in line with a prospective study on school-aged children and their parents after a paediatric medical event [27], we expected a positive relation between parental acute stress and child behaviour problems at 3 and 12 months postburn in mothers as well as in fathers, while controlling for child age, gender, and extent of the burn injury. Fourth, we explored parental worries about the child. We expected that both mother's and fathers' responses would include worries related to the burn (event). Fifth, differences within couples regarding worries about the child were explored. Finally, we explored the relation between parental acute stress and subsequent parental worries about the child at 3 and 12 months postburn.

\section{Methods}

\section{Procedure}

This study is part of a larger prospective study on child and parental adjustment following a paediatric burn event. Data were collected in three Dutch and four Belgian burn centres. In previous articles, we reported on acute stress and the course of parental stress symptoms in this sample [21, 26]. Data for the current study concerning child behavioural and emotional problems were collected at 3 months (T1) and 12 months postburn (T2). Parent acute subjective distress, a predictor variable, was collected within the first month postburn.

Families were eligible to participate if the child was between 8 months and 5 years old, length of stay in the hospital was $\geq 24 \mathrm{~h}$, and the total body surface area (TBSA) burned was $\geq 1 \%$. TBSA is a percentage used to express the estimated proportion of the body with second or third degree burns. Exclusion criteria were insufficient parental Dutch language proficiency and mental retardation in the child. Families were included between October 2007 and July 2010. A local researcher contacted eligible families 1-4 weeks after admission. The researcher explained the study purpose and offered additional written information; informed consent was obtained from each of the participants. Two independent ethics committees in the Netherlands and Belgium, respectively, approved this study.

Of 313 families that met the study criteria, we could use data of 155 families that completed at least one questionnaire on behaviour problems either at 3 or at 12 months postburn. Sixty-one families gave their informed consent to participate, but dropped out before 3 months postburn, 55 families declined the invitation, 26 families were missed before they could be approached, and 16 families were not invited because their participation was deemed inappropriate (e.g. severely ill family members, psychiatric background, or court custody cases). The 155 participating families did not differ from the other eligible families $(n=158)$ in terms of child age, gender, length of stay in the hospital, percentage TBSA, and percentage deep burns.

\section{Participants}

In total, 150 mothers and 125 fathers participated; in $77 \%$ of the cases, both parents participated. Table 1 shows child demographic and burn characteristics. Mean age of the mothers and fathers was 32.2 (SD 5.2) and 36.0 (SD 5.5) years. The majority of participating parents was employed (73\% of mothers, $93 \%$ of fathers), and had a partner (90\% of mothers, $95 \%$ of fathers).

In the course of the study, 25 families (23 mothers, 16 fathers) dropped out. We found no differences between families that participated at $\mathrm{T} 1$ and $\mathrm{T} 2$, and families that participated only at $\mathrm{T} 1$, in terms of child gender and age, burn characteristics, and mother report of child behaviour problems at T1. However, the 16 fathers that dropped out after $\mathrm{T} 1$ reported more $\mathrm{T} 1$ behaviour problems in their child, than the 95 fathers that participated at $\mathrm{T} 1$ and $\mathrm{T} 2$, $M=50.5 \quad(\mathrm{SD}=10.0) \quad$ vs. $\quad M=45.0 \quad(\mathrm{SD}=9.0)$, $t(109)=2.2, p=.03$. Finally, 16 families participated only at T2 (16 mothers and 14 fathers) because their child was $<18$ months at T1. Behaviour problems at T2 did not significantly differ between mothers/fathers that participated at T1 and T2 and mothers/fathers that participated only at $\mathrm{T} 2$.

Table 1 Child demographic and burn characteristics $(n=155)$

\begin{tabular}{llcl}
\hline & $M$ & SD & Range \\
\hline Child age (years) & 1.8 & 0.9 & $0.7-4.6$ \\
TBSA (\%) & 7.7 & 6.8 & $1-45$ \\
Length of stay in hospital (days) & 11.3 & 10.8 & $1-55$ \\
\hline & $n$ & $\%$ & \\
\hline Child gender (boys) & 101 & 65 & \\
Skin grafting procedure (yes) & 57 & 37 & \\
Site of accident & & & \\
Inside the home & 113 & 73 & \\
Somewhere else inside & 27 & 17 & \\
Outside & 15 & 10 & \\
Aetiology & & & \\
Hot fluid & 137 & 88 & \\
Hot object & 8 & 5 & \\
Flame & 7 & 5 & \\
Other & 3 & 2 & \\
\hline
\end{tabular}


Measures

\section{Child behaviour problems}

The Child Behavior Checklist (CBCL) $1 \frac{1}{2}-5$ is a 100 -item standardised measure of parental perception of their young child's emotional and behavioural problems [30]. For this study, both parents completed the validated Dutch version

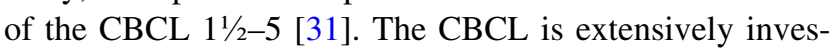
tigated and validity and reliability are excellent [30]. In this study, Cronbach's alphas for mother and father report of Internalising, Externalising and Total symptoms range between .79 and .94. Following the manual [30], scale scores were not computed if parents missed more than 8 out of 99 problem items. Missing items $(0.13 \%)$ for the other forms were substituted by zero (no problems).

Broad band scales 'Internalising problems', 'Externalising problems', and 'Total problems' were used as dependent variables in the analyses. Raw scores were transformed to $T$ scores, with possible scores ranging between 29 and 100 for Internalising problems and 28-100 for Externalising and Total problems (higher scores represent more problems). $T$ scores $60-63$ refer to the subclinical range, and scores $\geq 64$ to the clinical range of behaviour problems [30]. CBCL reference data were derived from the multicultural manual in which both the Netherlands and Belgium, together with 16 other countries including the United States of America, are represented in the medium-scoring group. This group consists of countries with mean total problem scores between 1 SD below and 1 SD above the 'omnicultural mean' [32].

\section{Parental worries}

Parental worries about the child were assessed through an open-ended CBCL question: 'What concerns you most about the child?'.

\section{Parental acute subjective distress}

The impact of event scale (IES) is a valid and psychometrically sound 15 -item self-report measure used to assess two dimensions of subjective distress related to traumatic events, i.e. symptoms of intrusion and avoidance [33, 34]. Both parents completed the validated Dutch version of the IES [35] within the first month postburn. Parents were asked to rate the frequency of symptoms they had experienced specifically in relation to their child's burn event on a 4-point Likert scale (0-1-3-5). The total possible score ranged from 0 to 75 , with higher scores representing higher levels of distress. Scores 26 and higher were used as an indication of clinically significant stress symptoms. Cronbach's alpha for the total IES was .86 for mothers and .87 for fathers, respectively.
Demographic and injury variables

Child gender, age, and burn characteristics (i.e. percentage TBSA, number of surgeries during initial hospitalisation, and length of stay in the hospital) were recorded from the medical file. Information regarding the cause of the burn, for example hot fluid, contact with a hot object, or flame, and the site of the accident was provided by parents within the first month postburn.

\section{Statistical analyses}

First, $T$ scores were used to represent child behaviour problems at each stage. Child behaviour problems in this sample were compared with published reference data by means of one-sample $t$ tests. Intraclass correlation coefficients (ICC's) were calculated to determine interparental agreement for child internalising and externalising behaviour problems. Reliability coefficients of $\geq .40$ reflect modest agreement, and ICC's of $\geq .75$ reflect strong agreement [36]. To assess interparental agreement for the categorical data on behavioural problems, the number of families was counted in which parents agreed on the absence or presence of behaviour problems in the (sub)clinical range.

Second, to interpret parental worries about the child, parents' answers on the open-ended CBCL question 'What concerns you most about the child?' were screened for overarching themes. After this initial screening, two researchers ( $\mathrm{AB}$ and $\mathrm{NVL}$ ) independently from each other categorised all parental answers into four themes: 1) physical aspects of burn injury recovery, 2) emotional and behavioural consequences of the burn injury, 3) concerns about the future and reactions from others related to the burn injury, and 4) 'general' developmental or behavioural issues not related to the burn. Across the respective stages, 224 parents described one or more concern. There was interrater consensus in $87 \%$ of the cases. In case of discrepancy, consensus was reached through discussion. Within-couple differences in terms of having or not having burn-related concerns and the relationship between acute parental distress (below or above cutoff) and the presence of subsequent burn-related concerns were tested with Chi-square statistics.

Third, the course and predictors of child behavioural problems were investigated in a multilevel regression model. Multilevel analysis can deal with multiple predictors in a dependent multilevel data structure. Our data set has a three-level hierarchy with time (3 and 12 months), nested within an observing parent (mother and father), nested within a child. An advantage of multilevel analysis concerns the ability to deal with missing data. Multilevel analysis considers all observations where information is available on the outcome level, using a missing at random assumption for the missing data [37]. 
Separate multilevel regression analyses for internalising and externalising problems were performed in MLwIN [38], using consecutive steps described by Hox [37]. First, intraclass correlations were calculated to examine which proportion of the variance in internalising and externalising problems was located at the time level (differences over time), parent level (differences between mother and father report), and child level (differences between children), respectively. Child gender, age, and burn severity were included in the model as covariates. To examine if mothers and fathers hold different views of their child's adjustment and to examine the influence of parental stress on parentreported child behavioural problems, parent gender and parent acute subjective distress were added to the model. An interaction term for 'parent gender' times 'parental distress' was tested to examine whether the influence of parental distress on child internalising and externalising symptoms differed between mothers and fathers. Finally, we checked whether the course of internalising and externalising symptoms differed between children ('random slopes'). This was evaluated by comparing the deviances of the models with and without the random parameter for time by means of Chi-square statistics. In case of random slopes, we explored if this randomness could be attributed to child gender, age, and extent of the burn injury by means of cross-level interactions between time and child factors. $T$ scores for internalising and externalising problems were used as dependent variables. All continuous independent variables (i.e. parent acute stress, child age, and burn size) were centred using the grand mean and dichotomous predictors (i.e. gender) were labelled 0-1.

\section{Results}

Prevalence of child behaviour problems

Parent ratings indicated that $9.0-15.7 \%$ of the children had behaviour problems in the (sub)clinical range at 3 months and 5.5-13.4\% of children had behaviour problems in the (sub)clinical range at 12 months after the burn event (Table 2). Behavioural problem scores were significantly lower than published reference data [32]. This applied to internalising, externalising, and total problems, at both 3 and 12 months postburn, respectively (statistics not reported).

\section{Parental agreement on child behaviour problems}

Intraclass correlations between mother and father report for internalising symptoms were .59 and .58, and for externalising symptoms .67 and .68 , at 3 and 12 months, respectively. Multilevel regression analysis also showed that two parents had very similar observations of problem behaviour in their child (3\% of internalising and $1 \%$ of externalising symptoms' variance was located at the parent level). The level of agreement for the presence or absence of behaviour problems in the (sub)clinical range was 92 and $98 \%$ for internalising and 92 and $92 \%$ for externalising symptoms, at 3 and 12 months, respectively.

Relationship between parental acute distress and child behaviour problems

The major part of variance in child symptoms was found at the child level (56\% for internalising and $59 \%$ for externalising problems), and another large part of the variance occurred at the time level (41\% for internalising and $40 \%$ for externalising problems). This indicated that children's problem behaviour differed from each other and that children's scores changed over time.

For internalising problems, results showed that older children in the preschool range experienced more symptoms (Table 3). Parental acute stress symptoms $[M=27.1$ $(\mathrm{SD}=14.0)$ for mothers; $M=16.9 \quad(\mathrm{SD}=12.7)$ for fathers] were associated with internalising problems across the first year postburn, such that children of more acutely distressed parents were reported to have more internalising behavioural problems at 3 and 12 months later. This relationship between parental acute stress and child

Table 2 Child behavioural problems

\begin{tabular}{|c|c|c|c|c|c|c|c|c|c|c|c|c|}
\hline & \multicolumn{6}{|c|}{3 months postburn (T1) } & \multicolumn{6}{|c|}{12 months postburn (T2) } \\
\hline & \multicolumn{3}{|c|}{$\begin{array}{l}\text { Mother-report } \\
n=134\end{array}$} & \multicolumn{3}{|c|}{$\begin{array}{l}\text { Father-report } \\
n=111\end{array}$} & \multicolumn{3}{|c|}{$\begin{array}{l}\text { Mother-report } \\
n=127\end{array}$} & \multicolumn{3}{|c|}{$\begin{array}{l}\text { Father-report } \\
n=109\end{array}$} \\
\hline & M & SD & $\geq \mathrm{T} 60(\%)$ & M & SD & $\geq \mathrm{T} 60(\%)$ & M & SD & $\geq \mathrm{T} 60(\%)$ & M & SD & $\geq \mathrm{T} 60(\%)$ \\
\hline Internalising & 45.6 & 10.9 & 11.2 & 44.7 & 9.5 & 9.0 & 43.9 & 9.8 & 5.5 & 43.2 & 9.9 & 5.5 \\
\hline Externalising & 48.9 & 9.6 & 15.7 & 47.8 & 9.0 & 10.8 & 46.4 & 10.6 & 13.4 & 46.3 & 9.6 & 6.4 \\
\hline Total & 47.3 & 10.1 & 11.9 & 45.8 & 9.3 & 9.0 & 44.7 & 10.1 & 7.9 & 44.0 & 9.6 & 6.4 \\
\hline
\end{tabular}

Sample means were depicted with $T$ scores. Scores $\geq$ T60 indicate the subclinical and clinical range of behaviour problems. Of all T2 parents, $16 / 127$ mothers and 14/109 fathers did not participate at T1 
internalising symptoms was comparable for mothers and fathers. The relation between time and internalising behaviour problems was random (model deviance decreased with $14.2, p<.01$ ), indicating that not all children displayed the same decrease in behaviour problems. One child factor was identified that partly explained the different patterns over time. That is, girls tended to show more internalising problems (2.19 points higher than boys), but they also showed a greater decline in problems over time (a decrease of 3.58 points for girls versus a decrease of 0.16 points for boys).

For externalising problems, parental acute subjective distress was related to more child externalising problems (Table 3). Child factors age, gender, and burn extent were not significantly related to the level of externalising behavioural problems. Over time, there was a decrease in externalising problem behaviours which appeared random (model deviance decreased with 48.0, $p<.01$ ). However, in contrast to internalising problem behaviour, we could

Table 3 Relationship between parental acute distress and child internalising and externalising behaviour problems

\begin{tabular}{|c|c|c|c|c|}
\hline & \multicolumn{2}{|c|}{$\begin{array}{l}\text { Internalising } \\
\text { problems }\end{array}$} & \multicolumn{2}{|c|}{$\begin{array}{l}\text { Externalising } \\
\text { problems }\end{array}$} \\
\hline & B & SE & B & SE \\
\hline \multicolumn{5}{|l|}{ Time variables } \\
\hline Intercept & $45.29 * * *$ & 0.95 & $48.88 * * *$ & 0.98 \\
\hline Time & $-0.16^{\mathrm{c}}$ & 0.84 & $-1.17^{\mathrm{c}}$ & 0.87 \\
\hline \multicolumn{5}{|l|}{ Parent variables } \\
\hline Parent gender ${ }^{\mathrm{a}}$ & -1.23 & 0.74 & -0.57 & 0.70 \\
\hline Acute stress symptoms ${ }^{b}$ & $0.14 * *$ & 0.05 & $0.09 *$ & 0.05 \\
\hline Parent gender $\times$ acute stress & 0.08 & 0.06 & 0.01 & 0.06 \\
\hline \multicolumn{5}{|l|}{ Child variables } \\
\hline Child gender ${ }^{\mathrm{a}}$ & 2.19 & 1.46 & 0.41 & 1.53 \\
\hline Child age & $2.59 * * *$ & 0.75 & 1.03 & 0.79 \\
\hline Burn size & 0.15 & 0.10 & 0.08 & 0.11 \\
\hline \multicolumn{5}{|l|}{ Interaction variables } \\
\hline Time $\times$ child gender & $-3.42 *$ & 1.49 & -2.39 & 1.55 \\
\hline Time $\times$ child age & -0.23 & 0.79 & -0.72 & 0.82 \\
\hline Time $\times$ child burn & -0.17 & 0.10 & -0.10 & 0.11 \\
\hline \multicolumn{5}{|l|}{ Explained variance } \\
\hline At the child level & \multicolumn{2}{|l|}{$28 \%$} & $\begin{array}{l}2 \% \\
\text { (of initial } 5\end{array}$ & $9 \%)$ \\
\hline Of random slopes ${ }^{c}$ & $12 \%$ & & $4 \%$ & \\
\hline
\end{tabular}

${ }^{\mathrm{a}} 0=$ male, $1=$ female

b Scale $0-75$

${ }^{c}$ Random at child level

$* p<.05$

$* * p<.01$

$* * * p<.001$ (two-sided tested) not find child factors that significantly contributed to the variance in children's courses of externalising symptoms.

Parental concerns

Between 33 and $57 \%$ of parents described one or more concerns with regard to their child at 3 and 12 months postburn, respectively. Table 4 shows the distribution of parental answers across the four themes: physical aspects of burn injury recovery, emotional and behavioural consequences of the burn injury, concerns about the future and reactions from others, and 'general' developmental or behavioural concerns. Examples of parental burn-related concerns were: ".. that the scar will remain visible" (physical aspects, mother, T1) "..will the skin grow along" (physical aspects, father, T2), “.. [my child] has imaginary friends since the burn..." (emotional or behavioural consequences, mother, T1), "how the outside world, future classmates will respond" (future and social aspects, father, $\mathrm{T} 2)$.

\section{Parental agreement on concerns}

Although a comparable proportion of mothers and fathers reported burn-related concerns, that is $31 \%$ at 3 months and $15-19 \%$ at 12 months, concurrent presence of concerns within couples was low ( 3 months: $X^{2}(1)=24.1$, $p<.01 ; 12$ months $\left.X^{2}(1)=17.2, p<.01\right)$.

Relationship between parental acute distress and parental concerns

Forty-seven percent of the mothers and $26 \%$ of the fathers had acute stress symptoms in the clinically significant range (IES $\geq 26$ ). Cross-tabs showed that the number of parents with burn-related concerns at 3 and 12 months postburn was approximately twice as high among highly symptomatic parents (Table 5). In contrast, symptomatic parents did not report more general behaviour concerns about their child (results not shown).

\section{Discussion}

By including both mother's and father's perspective on child internalising and externalising behaviour problems and both mother's and father's self-reported acute distress, this study provides a novel and comprehensive family perspective on child postburn adjustment. Results showed that overall child behavioural and emotional problems within the first year after the burn event were well within normal limits. However, $6-16 \%$ of the children were described to experience behaviour problems in the 
Table 4 Parental Concerns

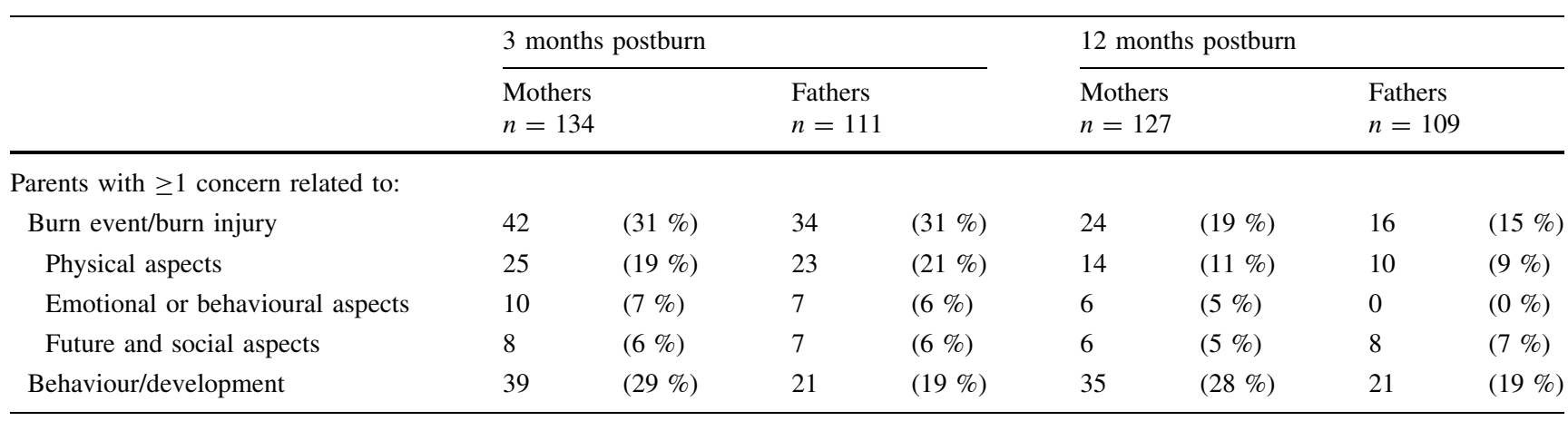

Each parent could describe more than one concern. Percentages in each cell are expressed as proportions of the total number of participating parents at the respective stages

Table 5 Relationship between parental acute distress and parental concerns

\begin{tabular}{lccc}
\hline & $\begin{array}{l}\text { Low acute parental } \\
\text { distress }(\%)\end{array}$ & $\begin{array}{l}\text { High acute parental } \\
\text { distress }(\%)\end{array}$ & $X^{2}$ \\
\hline Proportion of mothers with $\geq 1$ & burn-related concern & \\
3 months & 23 & 39 & $4.3^{*}$ \\
12 months & 14 & 26 & $2.7^{\dagger}$ \\
Proportion of fathers with $\geq 1$ burn-related concern & \\
3 months $\quad 24$ & 45 & $4.3^{*}$ \\
12 months $\quad 10$ & 31 & $7.1^{* *}$ \\
$* p<.05$ & & \\
$* * p<.01$ & & \\
$+p<.10$ & &
\end{tabular}

(sub)clinical range. In addition, a substantial proportion of parents reported concerns related to physical and emotional aspects of their child's injury. The findings further indicated an association between mothers' and fathers' own distress shortly after the burn event and child adjustment and parental worries across the first year after the burn event. The results point out the importance of incorporating a family systems perspective when supporting, educating, and monitoring families after a paediatric burn event.

Although a small decrease in children's symptoms over the first year was noticed, children in our sample actually displayed less internalising and externalising behavioural problems at either time point after the burn event in comparison to multinational reference data. To note, mothers and fathers held generally comparable views of their child's adjustment, which was consistent with our hypothesis. Possibly, sample and study characteristics may have contributed to the favourable outcome of behaviour problems in this study. In comparison to a US study that found elevated levels of behaviour problems in children with severe burns that came from families with a low socioeconomic background and of which many cases were suspected child abuse, our study, like other European studies [11, 14], predominantly included children with minor to moderate burns that came from families with a good socioeconomic background and of whom information regarding abuse was not known. Further, it is possible that children have experienced behaviour or emotional difficulties during and shortly after hospitalisation, but this would not have been captured in our first assessment of behaviour problems scheduled at 3 months postburn. Finally, although the current findings suggest a generally favourable behavioural outcome for young children with burns, results from recent diagnostic interview studies with parents have shown considerable rates of posttraumatic stress disorder and for instance oppositional defiant disorder in very young children with burns $[6,10,11]$. Perhaps, clinical parent interviews that focus on posttraumatic stress responses, such as intense psychological distress at cues related to the event, avoidance of stimuli related to the trauma, or sleep problems, are more sensitive to detect particular problematic domains for these young burn survivors. To note, externalising problems were in our study more prevalent than internalising problems, which may be due to the difficulties for parents to identify internalising problems in their young child.

The participation of fathers in this study provides additional insights in postburn mechanisms of family adjustment to a paediatric burn event, which may as well apply to other young trauma populations. In accordance with our hypothesis, child behaviour problems at 3 and 12 months postburn, in particular internalising behavioural problems, were related to both mothers' and fathers' symptoms of acute stress. Moreover, the strength of the relationship between parental and child adjustment in our study was comparable in mother-child and father-child dyads. To our knowledge, this had not been reported before in preschool injured children. Our findings were consistent with a study on school-aged children and their parents after a medical traumatic event [27], but differed from a 
previous study that only found a relationship between mother-reported child and maternal adjustment after a paediatric burn event [17]. However, it should be mentioned that the father's perspective on child adjustment in that study was absent [11]. The literature describes different explanations concerning the interrelatedness between child and parent distress following trauma. First, it is possible that children and parents respond in a similar way to the same event. Shared factors related to the event and injury and a shared biological vulnerability might contribute to this phenomenon [39]. Moreover, it has been hypothesised that parents who are traumatised themselves may fall short in providing an emotionally safe environment and adequate parenting practices for the child resulting in poorer child recovery of trauma [40]. Scheeringa and Zeanah [40] hypothesised that behaviour of family members may exacerbate the responses of other family members. Apart from resemblance and potential dynamics within the family in terms of posttrauma reactions, it may be conceivable that parents who are more distressed themselves observe more behavioural and emotional problems in their child. General child psychopathology literature for instance reported that parental characteristics, such as depression [41, 42] and anxiety [41], may influence parent ratings of psychopathology in the child. After medical trauma, it was found that highly distressed parents tended to overestimate traumatic stress symptoms in their school-aged child, whereas less distressed parents actually underestimated their child's symptoms in comparison to the child's self-report [43]. To what extent parental distress may have influenced the parent's observation of their child remains to be elucidated in future research.

This study identified two child factors that were associated with postburn internalising behavioural problems. Internalising symptoms were more strongly present in older children in the preschool age range, while age differences concerning internalising symptoms in the general population of 1.5- to 5-year-olds are minimal [30] and previous burn studies with preschool children did not find an association between child age and postburn total behaviour problems [11, 14]. Developmental factors may influence particular important aspects of the experience of a traumatic event, such as comprehension of what is happening, prior knowledge, and emotion regulation [44]. These factors may have been less present in the youngest children in this study, perhaps resulting in less internalising responses in this group. Another explanation may be that these symptoms are better observable in toddlers compared to infants, resulting in higher scores in older children. Related to this issue, the measure used in this study, albeit specifically designed for 1.5- to 5-year-olds, is perhaps more sensitive to detect behavioural changes in the older age range of preschool children. With respect to the decrease of internalising symptoms, results showed that this pattern was different for boys and girls. Where girls tended to show more overall internalising problems than boys, they also showed a greater decrease in symptoms. Although one study reported that young traumatised girls exhibited somewhat more PTSD symptoms than boys [45], overall there seems no consistent evidence for gender differences regarding trauma reactions in preschool traumatised children [40]. The CBCL manual reports no general gender effects [30]. In contrast to internalising symptoms, the variables under study explained only a minor part of externalising symptoms and we could not find specific factors that contributed to the decrease in externalising symptoms over time. Other factors beyond the scope of this study, such as family functioning [11], parenting behaviour [40], and, although speculative, parent's posttraumatic symptoms of hyperarousal after the burn event, might have contributed stronger to the child's externalising symptoms.

A substantial group of mothers and fathers in this study expressed concerns about their child's adjustment to the burn trauma, relating to physical, social, and emotional aspects. This is in line with a qualitative study among family members of paediatric patients with burns that found many burn-related concerns already shortly after hospitalisation [7]. However, specific reports about father's worries had not been described before. The content of parental worries suggests a need for comprehensive psycho-education for parents about physical as well as emotional aspects of wound healing and scar formation as an essential part of postburn aftercare. Although mothers and fathers within couples had quite comparable views of their child's behaviour problems, parents did not report the same burn-related worries about their child. As we found a clear relation between parents' own distress and their subsequent burn-related worries, the low concordance on worries may partly be explained by the low concordance on traumatic stress within parent couples that we previously found [21]. Awareness about the relationship between parents' stress reactions in the subacute phase of the injury and long-term worries and questions about the child's recovery is warranted and the needs of both parents in this perspective should be an on-going focus of monitoring.

This prospective multicentre study has a number of important strengths, including a longitudinal design and a large sample of preschool children with burns. We used a standardised and well-validated instrument to examine child behavioural and emotional problems from both the mother's and the father's perspective, and advanced statistical modelling to adequately study family data over time. Limitations of this study include the lack of information on pre-injury behavioural functioning that prevented to examine a causal relationship between the burn 
event and postburn child problems. In other studies after accidental trauma [13, 46], pretrauma behaviour was strongly linked to behaviour after traumatic injuries. In general, it is difficult to gather reliable (parent-reported) information concerning child pre-injury behaviour problems once admitted to the hospital, but it should nevertheless be a point of attention. Future studies may consider a comparison or control group to assess the unique consequences of paediatric burns as well as to gain more insight in recovery processes. In addition, the role of parental traumatic stress symptoms specifically related to the child's burn event was investigated in this study, but other parental stressors may as well have played a role. For example, Meyer and colleagues [47] found that parents attributed their perceived stress more often to child characteristics than to parent characteristics. Concerning the prevalence rate of (sub)clinical problem behaviour, we used reference data from a multicultural, but US-based, normative sample [32]. No separate norm data are available for Belgium and the Netherlands. It is remarkable that the prevalence rates we found in this study were much more in line with a sample of Swiss preschool children with burns [11], than with published norms. Notably, five percent of eligible children were excluded because of problematic family conditions, and fathers that dropped out in the course of the study indicated slightly more problem behaviour in their child, so prevalence rates of behaviour problems might actually be higher. With regard to the population under study, insufficient proficiency in the Dutch language was an exclusion criterion, though these families were well represented in the paediatric burn population. Future studies may benefit from a longer follow-up than 1 year, as one study identified burn-related factors to be associated with maternal symptoms of traumatic stress over a 10-year period [48], raising questions to what extent these factors might affect the child's long-term functioning. Finally, research on family dynamics should give more insight how parents' reactions and young children's behaviour reciprocally influence each other in the aftermath of a medical traumatic event.

Results from this study may inform European clinical burn practice where young children-predominantly with scald burns-and their parents are frequently seen. Our findings showed that parents hold fairly comparable views of their child's behaviour following the burn event. Health-care workers should be aware that, as in the normal population, a subgroup of children presents with clinical behaviour problems. Parents' concerns about their child's burn, which were related to physical, emotional, and potential future social aspects of their child's injury, provide suggestions for several important themes for psycho-education materials. In addition, parents within couples did not express the same concerns, indicating the importance of enquiring after concerns of mother as well as of fathers. Parents may struggle with intense emotions or traumatic stress reactions, which may also relate to (their observation of) their child's behaviour problems and to parental worries about the child. Information about a range of normative parental reactions to stressful events may be helpful and supporting for both parents. Finally, considering the interrelatedness between the child and both parents in terms of postburn outcome, we recommend a broad family perspective on child adjustment and aftercare in the aftermath of a paediatric medical traumatic event.

Acknowledgments The Dutch Burns Foundation funded this study (grant number 07.107). This work resulted from collaborative efforts from our research team (M. Bremer, G. Bakker, A. Boekelaar, A. van de Steenoven, H. Hofland, L. Braem, A. Goemanne, H. Hendrickx; K. Maertens) in the respective burn centres in the Netherlands (Groningen, Beverwijk, Rotterdam) and Belgium (Antwerp, Neder-overHeembeek, Gent, Leuven). We thank all parents for their participation.

Conflict of interest The authors declare that they have no conflict of interest.

\section{References}

1. Stoddard FJ, Saxe G (2001) Ten-year research review of physical injuries. J Am Acad Child Adolesc Psychiatry 40(10):1128-1145

2. Vloemans AF, Dokter J, van Baar ME, Nijhuis I, Beerthuizen GI, Nieuwenhuis MK, Kuijper EC, Middelkoop EM (2011) Epidemiology of children admitted to the Dutch burn centres. Changes in referral influence admittance rates in burn centres. Burns 37(7):1161-1167

3. Brusselaers N, Monstrey S, Vogelaers D, Hoste E, Blot S (2010) Severe burn injury in Europe: a systematic review of the incidence, etiology, morbidity, and mortality. Crit Care 14(5):R188

4. Dissanaike S, Rahimi M (2009) Epidemiology of burn injuries: highlighting cultural and socio-demographic aspects. Int Rev Psychiatry 21(6):505-511

5. Landolt MA, Grubenmann S, Meuli M (2002) Family impact greatest: predictors of quality of life and psychological adjustment in pediatric burn survivors. J Trauma 53(6):1146-1151

6. De Young AC, Kenardy JA, Cobham VE, Kimble R (2012) Prevalence, comorbidity and course of trauma reactions in young burn-injured children. J Child Psychol Psychiatry 53(1):56-63

7. Thompson R, Boyle D, Teel C, Wambach K, Cramer A (1999) A qualitative analysis of family member needs and concerns in the population of patients with burns. J Burn Care Rehabil 20(6):487-496

8. Kazak AE, Kassam-Adams N, Schneider S, Zelikovsky N, Alderfer MA, Rourke M (2006) An integrative model of pediatric medical traumatic stress. J Pediatr Psychol 31(4):343-355

9. Mason SA, Hillier VF (1993) Young, scarred children and their mothers-a short-term investigation into the practical, psychological and social implications of thermal injury to the preschool child. Part II: implications for the child. Burns 19(6):501-506

10. Stoddard FJ, Saxe G, Ronfeldt H, Drake JE, Burns J, Edgren C, Sheridan R (2006) Acute stress symptoms in young children with burns. J Am Acad Child Adolesc Psychiatry 45(1):87-93 
11. Graf A, Schiestl C, Landolt MA (2011) Posttraumatic stress and behavior problems in infants and toddlers with burns. J Pediatr Psychol 36(8):923-931

12. Liber JM, List D, Van Loey NE, Kef S (2006) Internalizing problem behavior and family environment of children with burns: a Dutch pilot study. Burns 32(2):165-171

13. Meyer WJ, Robert R, Murphy L, Blakeney PE (2000) Evaluating the psychosocial adjustment of 2- and 3-year-old pediatric burn survivors. J Burn Care Rehabil 21(2):179-184

14. Kent L, King H, Cochrane R (2000) Maternal and child psychological sequelae in paediatric burn injuries. Burns 26(4): 317-322

15. Bakker A, Maertens K, Van Son MJM, Van Loey NEE (2013) Psychological consequences of pediatric burns from a child and family perspective: a review of the empirical literature. Clin Psychol Rev 33(3):361-371

16. Moreno J, Silverman WK, Saavedra LM, Phares V (2008) Fathers' ratings in the assessment of their child's anxiety symptoms: a comparison to mothers' ratings and their associations with paternal symptomatology. J Fam Psychol 22(6):915-919

17. Duhig AM, Renk K, Epstein MK, Phares V (2000) Interparental agreement on internalizing, externalizing, and total behavior problems: a meta-analysis. Clin Psychol Sci Prac 7(4):435-453

18. Scheeringa MS, Zeanah CH (2001) A relational perspective on PTSD in early childhood. J Trauma Stress 14(4):799-815

19. El Hamaoui Y, Yaalaoui S, Chihabeddine K, Boukind E, Moussaoui D (2006) Depression in mothers of burned children. Arch Womens Ment Health 9(3):117-119

20. Phillips C, Rumsey N (2008) Considerations for the provision of psychosocial services for families following paediatric burn injury-a quantitative study. Burns 34(1):56-62

21. Bakker A, Van Loey NE, Van der Heijden PG, Van Son MJ (2012) Acute stress reactions in couples after a burn event to their young child. J Pediatr Psychol 37(10):1127-1135

22. McGarry S, Girdler S, McDonald A, Valentine J, Wood F, Elliott C (2013) Paediatric medical trauma: the impact on parents of burn survivors. Burns 39:1114-1121

23. Hall E, Saxe G, Stoddard F, Kaplow J, Koenen K, Chawla N, Lopez C, King L, King D (2006) Posttraumatic stress symptoms in parents of children with acute burns. J Pediatr Psychol 31(4):403-412

24. Rizzone LP, Stoddard FJ, Murphy JM, Kruger LJ (1994) Posttraumatic stress disorder in mothers of children and adolescents with burns. J Burn Care Rehabil 15(2):158-163

25. Mason SA, Hillier VF (1993) Young, scarred children and their mothers-a short-term investigation into the practical, psychological and social implications of thermal injury to the preschool child. Part III: factors influencing outcome responses. Burns 19(6):507-510

26. Bakker A, Van Son MJM, Van der Heijden PGM, Van Loey NEE (2013) Course of traumatic stress reactions in couples after a burn event to their young child. Health Psychol 32(10):1076-1083

27. Landolt MA, Ystrom E, Sennhauser FH, Gnehm HE, Vollrath ME (2012) The mutual prospective influence of child and parental post-traumatic stress symptoms in pediatric patients. J Child Psychol Psychiatry 53(7):767-774

28. Morris A, Gabert-Quillen C, Delahanty D (2012) The association between parent PTSD/depression symptoms and child PTSD symptoms: a meta-analysis. J Pediatr Psychol 37(10):1076-1088

29. Connell AM, Goodman SH (2002) The association between psychopathology in fathers versus mothers and children's internalizing and externalizing behavior problems: a meta-analysis. Psychol Bull 128(5):746-773
30. Achenbach TM, Rescorla LA (2000) Manual for the ASEBA Preschool Forms \& Profiles. University of Vermont, Research Center for Children, Youth \& Families, Burlington, VT

31. Verhulst FC, Jvd Ende (2000) Nederlandse vertaling CBCL: Gedragsvragenlijst voor kinderen van 1 1/2-5 jaar [Dutch translation $\mathrm{CBCL}$. Erasmus $\mathrm{MC}$-Sophia Kinderziekenhuis, Rotterdam

32. Achenbach TM, Rescorla LA (2010) Multicultural Supplement to the Manual for the ASEBA Preschool Forms \& Profiles. University of Vermont, Research Center for Children, Youth, \& Families, Burlington, VT

33. Sundin EC, Horowitz MJ (2002) Impact of Event Scale: psychometric properties. Br J Psychiatry 180:205-209

34. Horowitz M, Wilner N, Alvarez W (1979) Impact of Event Scale: a measure of subjective stress. Psychosom Med 41(3):209-218

35. Brom D, Kleber RJ (1985) De Schok Verwerkings Lijst [The Impact of Event Scale]. Nederlands Tijdschrift voor de Psychologie 40:164-168

36. Streiner DL, Norman GR (2001) Health measurement scales, a practical guide to their development and use. Oxford University Press, Oxford

37. Hox JJ (2010) Multilevel Analysis, 2nd edn. Techniques and applications, Routledge

38. Rasbash J, Browne W, Goldstein H, Yang M, Plewis I, Healy M (2000) A user's guide to MLwiN. University of London, Multilevel Models Project

39. Langeland W, Olff M (2008) Psychobiology of posttraumatic stress disorder in pediatric injury patients: a review of the literature. Neurosci Biobehav Rev 32(1):161-174

40. De Young AC, Kenardy JA, Cobham VE (2011) Trauma in early childhood: a neglected population. Clin Child Fam Psychol Rev 14(3):231-250

41. De Los Reyes A, Kazdin AE (2005) Informant discrepancies in the assessment of childhood psychopathology: a critical review, theoretical framework, and recommendations for further study. Psychol Bull 131(4):483-509

42. Muller JM, Achtergarde S, Furniss T (2011) The influence of maternal psychopathology on ratings of child psychiatric symptoms: an SEM analysis on cross-informant agreement. Eur Child Adolesc Psychiatry 20(5):241-252

43. Kassam-Adams N, Garcia-Espana JF, Miller VA, Winston F (2006) Parent-child agreement regarding children's acute stress: the role of parent acute stress reactions. J Am Acad Child Adolesc Psychiatry 45(12):1485-1493

44. Salmon K, Bryant RA (2002) Posttraumatic stress disorder in children. The influence of developmental factors. Clin Psychol Rev 22(2):163-188

45. Green BL, Korol M, Grace MC, Vary MG, Leonard AC, Gleser GC, Smitson-Cohen S (1991) Children and disaster: age, gender, and parental effects on PTSD symptoms. J Am Acad Child Adolesc Psychiatry 30(6):945-951

46. Cox CM, Kenardy JA, Hendrikz JK (2008) A meta-analysis of risk factors that predict psychopathology following accidental trauma. J Spec Pediatr Nurs 13(2):98-110

47. Meyer WJ, Blakeney P, Moore P, Murphy L, Robson M, Herndon D (1994) Parental well-being and behavioral adjustment of pediatric survivors of burns. J Burn Care Rehabil 15(1):62-68

48. Bakker A, Van Loey NE, Van Son MJ, Van der Heijden PG (2010) Brief report: mothers' long-term posttraumatic stress symptoms following a burn event of their child. J Pediatr Psychol 35(6):656-661 